\title{
Transplantationen: Die Ursachen der Depression
}

\begin{abstract}
Auch nach einer hämatopoetischen Zelltransplantation (HCT) leiden zahlreiche Patienten unter Depressionen oder Fatigue. In einer größeren Studie wurde nun untersucht, welche Faktoren die Gefahr erhöhen.
\end{abstract}

epressionen und Fatigue gehören zu den beiden häufigsten Symptomen, von denen Patienten während und nach einer HCT berichten. Dabei beeinträchtigen sie nicht nur die Lebensqualität, sondern gehen auch mit einer ungünstigen Prognose einher. So haben die Patienten z. B. ein erhöhtes Mortalitätsrisiko. Heather S. L. Jim und Kollegen suchten nach demografischen und sozialen Risikofaktoren für das Auftreten von Depressionen und Fatigue in einer großen Kohorte von HCT-Überlebenden. Bei den Patienten wurden im Rahmen der Nachsorge jährlich Gesundheitsinformationen erhoben, in die auch soziodemografische Fragen einflossen. Die kli- nischen Charakteristika der Patienten stammten aus den Krankenakten.

Es nahmen 1.869 HCT-Empfänger an der Studie teil. Sie waren im Median 56 Jahre alt, $53 \%$ von ihnen waren männlich. Im Mittel lag die HCT 13 Jahre zurück, wenn es sich um eine allogene Spende (alloHCT) handelte; bei autologen Zellen waren es 6 Jahre. Unter moderater bis schwerer Depression litten $13 \%$ der Teilnehmer, unter einer moderaten bis schweren Fatigue $42 \%$. Unabhängig mit einem höheren Depressionsrisiko assoziiert $(\mathrm{p}<0,01)$ waren unter den alloHCT-Empfängern weibliches Geschlecht, jüngeres Alter, chronische Schmerzen zum Abfragezeitpunkt und die von den Patienten berichtete Schwere der chronischen Graft-versus-Host-Disease (GvHD). Mit einem größeren Fatigue-Risiko waren weibliches Geschlecht, aktuelle chronische Schmerzen und die aktuell berichtete Schwere der GvHD unabhängig assoziiert $(\mathrm{p}<0,01)$. Unter den Empfängern einer autologen HCT waren jüngeres Alter sowie aktuell auftretende chronische Schmerzen unabhängig mit einem höheren Risiko für Depressionen und für $\mathrm{Fa}$ tigue assoziiert $(\mathrm{p}<0,01)$.

Fazit: Depressionen und Fatigue sind bei Langzeitüberlebenden nach HCT eine häufige Belastung. Vor allem soziodemografische Faktoren scheinen entscheidend für das Auftreten zu sein. Es bedarf eines besseren Screenings und gezielter Interventionen.

Christina Berndt

Jim HSL et al. Risk Factors for Depression and Fatigue Among Survivors of Hematopoietic Cell Transplantation. Cancer. 2016;122(8):1290-7.

\section{Hier steht eine Anzeige.}

\section{Springer}

